\title{
Review
}

\section{Gut Microbiota: The Servant of Human Being and the Accessary of Tumorigenesis}

\author{
Pan $\mathrm{Gu}^{1,2}, \mathrm{Di}^{\mathrm{Li}}{ }^{2}$, Gang $\mathrm{Xu}^{1,3}$, Yingnan $\mathrm{Sun}^{1 \star}$, Minghua $\mathrm{Wu}^{1,2 \star}$ \\ 1. Hunan Cancer Hospital and the Affiliated Cancer Hospital of Xiangya School of Medicine, Central South University, \\ Changsha 410013, Hunan, China. \\ 2. The Key Laboratory of Carcinogenesis of the Chinese Ministry of Health, The Key Laboratory of Carcinogenesis and \\ Cancer Invasion of the Chinese Ministry of Education, Cancer Research Institute, Central South University, Changsha, Hunan \\ 410008, China. \\ 3. The Hengyang Medical School, University of South China, Hengyang, Hunan, 421001, China.
}

*Corresponding authors: Yingnan Sun, Email: sunyingnan29@126.com; Minghua Wu, Email: wuminghua554@aliyun.com

\section{Abstract}

Gut microbiome affects multiple facets of human health and is inextricably linked to tumorigenesis. Substantial research has aimed to understand how gut microbiome functions in the homeostasis of our body. This review explores the evidence demonstrating how the gut microbiome may affect body health, thereby having an impact on metabolism, protection, nutrition and some anatomical-functional relationship. Alterations in gut microbiota composition have been associated with plenty disorders. Of interest, majority of researches demonstrate the role of microbiota in cancer. However, they haven't been classified systematically. We divided them into tumor in situ, gut-biliary tract cancer axis, gut-breast cancer axis, gut-haematopoiesis cancer axis and gut-brain cancer axis. In addition, we introduce the latest method in diagnosis and treatment of related cancer.

Keywords

Gut microbiome; Function; Cancer; Axis; Diagnosis; Treatment

\section{INTRODUCTION}

The human body is a nutritious culture medium, supporting the growth of a wide variety of microbial species $^{[1]}$. There has been estimated that $10^{[14]}$ microorganisms reside in various parts of the human body including

(1) The Author(s) 2022. Open Access This article is licensed under a Creative Commons Attribution 4.0 International License (https://creativecommons.org/licenses/by/4.0/), which permits unrestricted use, sharing, adaptation, distribution and reproduction in any medium or format, for any purpose, even commercially, as long as you give appropriate credit to the original author(s) and the source, provide a link to the Creative Commons license, and indicate if changes were made. 
skin surfaces and body cavities, among which the intestinal tract possess the major taxonomic units and genetic contents $^{[2]}$. It is also estimated that the number of bacteria we carry reach 1011 per gram of luminal content $t^{[3]}$. Microbiome defined as a community of microorganisms especially indicate those living in or on the human body, while here, we mainly focused on the gut microbiota, the microbial community in digestive tract involving stomach, small intestine, and large intestine ${ }^{[4]}$.

Gut microbiota could be easily affected by environmental changes (infection, diet or lifestyle), resulting in symbiotic imbalance and disease. Evidence in mounting numbers indicates gut microbiota play a key role in carcinogenesis. As we all known, hallmarks of cancer are comprised of eight biological capabilities such as sustained proliferation, growth suppression evasion, tissue invasion and metastasis, replicative immortality, angiogenesis induction, cell death resistance, altered cellular energetics, and immune evasion ${ }^{[5]}$. Conceptual progress recently has added more emerging hallmarks of potential generality to previous list like escape of circadian regulation ${ }^{[6]}$, development of genomic instability and inflammatory state of premalignant and frankly malignant lesions. Nevertheless, gut microbiota may contribute to all above the tumor hallmarks.

\section{WHAT'S THE NORMAL FUNCTION OF GUT MICROBIOTA?}

With the development of high throughput metagenomic gene sequencing technology, more and more researches are being increasingly recognized that gut microbiota exceeds human genome in number and has close interaction with human homeostasis, maintaining health ${ }^{[7,8]}$.

The main known functions of the gut microbiota can be broadly classified into three groups: metabolic group functioned in digestion of food, protective group functioned in protecting against pathogens, and trophic group functioned in controlling proliferation and differentiation of epithelial cells.

\section{METABOLISM}

Gut microbiota has essential influence on energy harvest from the dietary carbohydrates and energy reservation. The main mechanism are hydrolysis and subsequent fermentation. The microbial metabolite indole promotes barrier function through the pregnane $\mathrm{X}$ receptor ${ }^{[9]}$. Colonic organisms such as Bacteroides, Roseburia, Bifidobacterium, Fecal bacterium, and Enterobacteria generate short chain fatty acids (SCFA) through fermentation of the carbohydrates, supplying plenty energy ${ }^{[10,11]}$. The SCFAs accompanied Peptide Tyrosine Tyrosine/Pancreatic Peptide YY3-36 (PYY) reversely reduce appetite and alter the energy metabolism to keep $\mathrm{fit}^{[12,13]}$. Furthermore, Oxalobacter formigenes, Lactobacillus, and Bifidobacterium prevent the synthesis of oxalate to reduce the risk of kidney stone formation ${ }^{[14]}$. Amino acids (AA) decomposed from diet is significant in maintaining functional intestinal mucosal ${ }^{[15]}$. It can also directly incorporate into bacterial cells, involved in latter synthesis and bioavailability ${ }^{[16]}$. For instance, Lhistidine and glutamate can be converted to histamine and G-amino butyric acid (GABA) respectively by the bacterial enzyme ${ }^{[17,18]}$. When it comes to plant-derived products, gut microbiota is also participated in metabolism of polyphenols, microbial-derived phenolic acids, which is characterized by bioactive potential like antioxidant, anticarcinogenic, anti-inflammatory and neuroprotective ${ }^{[19]}$. Researchers found that some polyphenols can cross the blood brain barrier and reach the brain in a significant concentration and block the formation of amyloid aggregates, performing the modulatory effect on Alzheimer's disease $^{[20]}$. Reversely, polyphenols can also modulate the composition of gut microbiota to reach a balance ${ }^{[21,22]}$. Gut microbiota regulate the host's lipid metabolism mainly through (i) variation of lipoprotein cholesterol levels ${ }^{[23]}$, (ii) synthesis of secondary bile acids ${ }^{[24]}$, (iii) modulation of endocannabinoid system $^{[25]}$, (iv) LPS- mediated inflammation $^{[26]}$, (v) inhibition of lipoprotein lipase activity in adipocytes ${ }^{[27]}$. Similarly, lipid can also regulate gut microbiota composition. Polyunsaturated fatty acids accumulation will result in a decrease in Faecalibacterium, and increase in Bacteroidetes and butyrate-producing bacteria ${ }^{[28]}$. As to other metabolism, an interesting example is 
drug metabolism that the microbial $\beta$ glucoronidase will cause deconjugation of the anticancer drug irinotecan and lead to diarrhea, inflammation and anorexia ${ }^{[29]}$.

\section{PROTECTION}

In order to protect host against colonization by pathogens, gut microbiota cooperates with immune systems ${ }^{[30]}$. Innate immune system can sense microorganisms at once and the metabolic products functioned as signal will induce quick responses ${ }^{[31]}$. The intestinal mucus layer is able to keep mutualism by keeping bacteria at bay and restricting overt immune stimulation ${ }^{[32]}$. Researchers found that gut microbiota play an important role in remodeling intestinal immune microenvironment. Firstly, the level of myelopoiesis as well as microglia correlates with the complexity of the gut microbiota ${ }^{[33]}$, which might be regulated by $\mathrm{SCFAs}^{[34]}$. In addition, gut microbiota also controls the transportation of those cells in the gut and replenish monocytes in the intestinal mucosa through expressing C-C chemokine receptor type 2 (CCR2 $)^{[35]}$. Apart from innate immune cells, lymphoid cells can only function well with microbial colonization ${ }^{[36]}$. Lymphotoxin- $\alpha$ produced by lymphoid cells is crucial for the generation of $\operatorname{IgA}^{[37]}$. IL-22 secreted by lymphoid cells will induce expression of the enzyme fucosyltransferase 2 and fucosylation of surface proteins by intestinal epithelial cells, which is required for host defense against enteric pathogens $^{[23]}$.

\section{NUTRIENT}

Gut microbiota-derived SCFAs are energy source for the epithelium. What's more, SCFAs can strengthen epithelial barrier through oxygen consumption and hypoxia-inducible factor ${ }^{[38]}$. Butyrate, which considered as the strongest effect SCFA, could modify and repair the microstructure of the small and large intestine, accelerating intestinal mucosa maturation during the development. Hence, through regulate gene expression and protein synthesis, SCFAs possess the ability to enhance proliferation, differentiation, and maturation and reduces apoptosis of normal enterocytes in the small intestine. In colon, the absence of SCFAs induces massive apoptosis in colonocytes. For example, SCFAs might not the only energy source for these cells, but the indirect effect of SCFAs including stimulation of gastrointestinal peptides and growth factors releasing may play a key role in cell proliferation $^{[39]}$.

\section{OTHER FUNCTIONS}

There is a close anatomical and functional relationship between the intestinal tract and the liver which is known as gut-liver axis, which was found to be a major reason in the development and progression of nonalcoholic fatty liver disease (NAFLD) and obesity. This may attribute to molecules generated by abnormal gut microbiome (overgrowth of small intestinal bacteria, intestinal dysbiosis and increased permeability of intestinal) could be transferred to the liver. As the incidence of NAFLD is increasing, gut-liver axis is treated as a promising target for NAFLD therapy ${ }^{[40]}$. In addition to the gut-liver axis, the enteric nervous system is another investigational focus. The brain nervous system development might be affected in the absence of a gut microbiota compared with normal animals ${ }^{[41]}$, indicating that the importance of long-lasting effects of appropriate microbial colonization. Gut microbiota is reported with the ability to produce neuroactive metabolites, such as SCFA and ketone bodies ${ }^{[42,43]}$; phenolic acids ${ }^{[44,45]}$; steroids ${ }^{[46]}$; AAs and their derivatives ${ }^{[47,48]}$; catecholamines ${ }^{[49]}$. Such metabolites functioned through direct interaction with intestinal tract's receptors and diffusion effect to stimulate neurophysiology ${ }^{[50]}$. Indeed, huge array of biologically active substances could be produced by gut microbiota to influence motility and other function of intestinal tract ${ }^{[51]}$. What's more, investigators proved gut microbiota also influence cerebral metabolites through the microbiota-gut-brain axis. People with normal gut microbiota are more likely gain brain health ${ }^{[52]}$, which is helpful in discovery of newly therapeutic target for some neurological disorders like stressinduced pathological conditions ${ }^{[53]}$. 


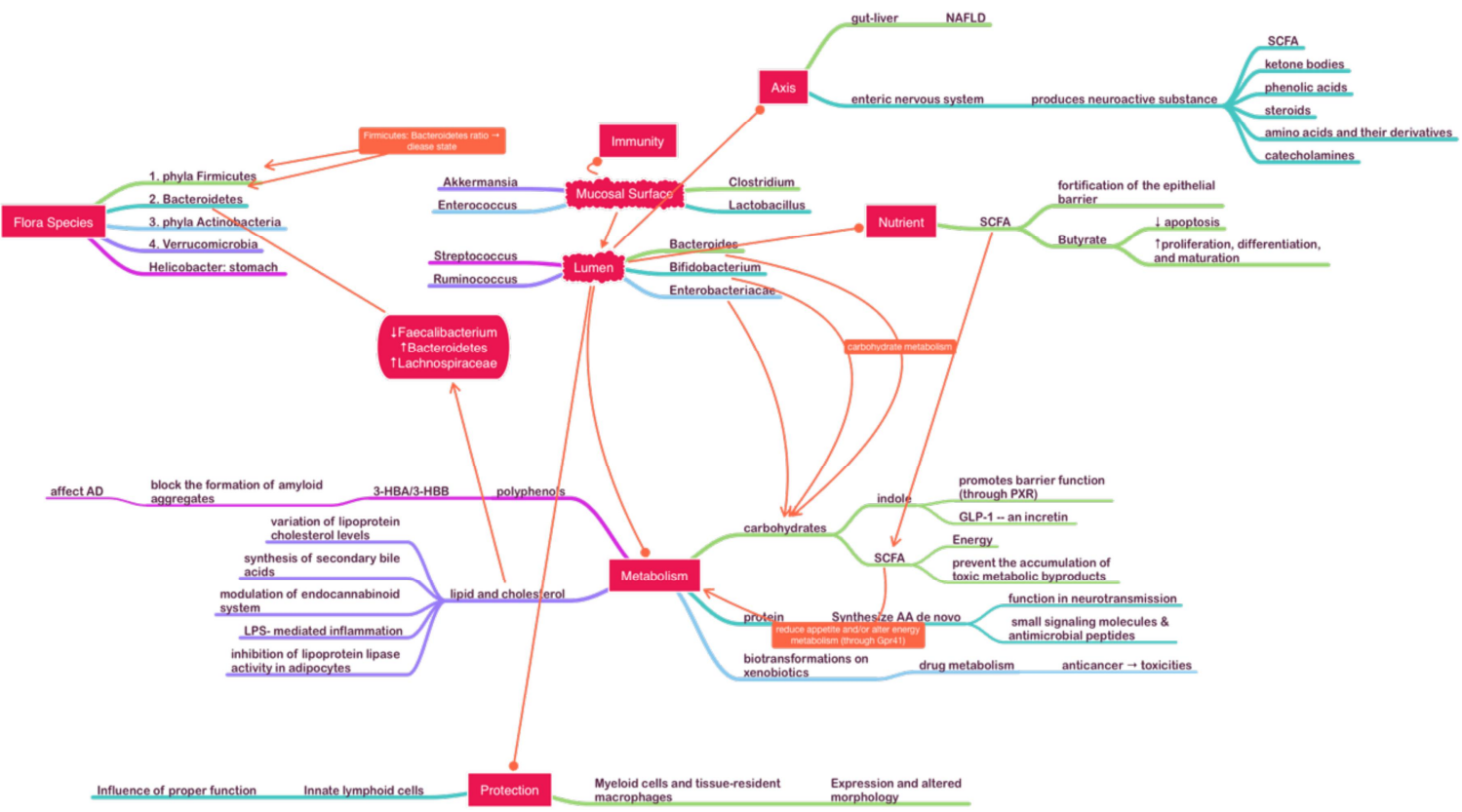

Fig 1 Normal Function of Gut Microbiota

\section{HOW GUT MICROBIOTA INDUCE CANCER?}

As what we mentioned above, gut microbiota plays an important role in maintaining mental and physical health ${ }^{[54]}$. Although we can depict how some bacteria servant in physiological activities, the role of most bacterial species in health and disease remains largely unknown ${ }^{[55]}$. Colorectal cancer burden (CRC) was substantially increase since some unhealth lifestyle was adapted ${ }^{[56]}$. Factors like consumption of foods, lifestyle, genetic polymorphisms and aging affect not only microbiota composition but also cancer susceptibility ${ }^{[57-59]}$. Some studies have supported specific bacterial agents participant in tumorigenesis including Helicobacter pylori (H. pylori) ${ }^{[60]}$ and human papillomavirus $^{[61]}$. Herein, we discuss how it work through host-microbe, microbe-microbe, and environmental interactions in both colorectal cancer and non-intestinal cancers.

\subsection{Colorectal cancer}

As a common malignancy in the world, CRC accompanied with the adoption of western lifestyle in recent years in China, the 5-year prevalence proportion has reached $74.6-58.3$ per $100,000^{[62]}$. Data in China was updated that more than 376, 000 new CRC patients and 191,000 deaths occur every year ${ }^{[63]}$. According to the epidemiological studies, several lifestyle factors that affect the risk for developing CRC. Ranging from enteric fungal microbiota to bacterial, can contribute to CRC with commensal system break up ${ }^{[64]}$. The way that gut microbiota induce CRC can be summarized as the following.

\subsubsection{Chronic inflammation drives carcinogenesis and sustain a pro-inflammatory microenvironment}

Chronic inflammation drives carcinogenesis was widely established in the long run. However, it has been verified recently in molecular biological level. Numerous genes were found altered through metagenomic analysis among the healthy, adenoma, and CRC patients ${ }^{[65]}$. Further analysis discovered that patients with CRC have high level of red meat consumption associated with highly expressed C-reaction protein. What's more some bacterial metabolites were different among groups. It is identified that Fusobacterium, Parvimonas, Gemella, and Leptotrichia were enriched in early-stage colorectal cancer and the abundance of anti-inflammatory F. prausnitzii were lost ${ }^{[66]}$, inducing inflammation in intestinal tract which also promoted the change of microbiome community and facilitate bacterial translocation into neoplastic tissue. The cumulative effect will further enhance 
the expression of inflammatory cytokines and lead to tumor formation ${ }^{[67]}$. Gut microbiota could also sustain a proinflammatory microenvironment through recruitment of immune cells to drive carcinogenesis. ${ }^{[68]}$

\subsubsection{Direct ways and indirect ways to elicit DNA damage and alter gene expression}

Some bacteria can promote carcinogenesis in a direct way to elicit DNA damage. For example, E.coli was proved to express Colibactin gene, inducing DNA damage, chromosome aberrations and increased gene mutations in vivo ${ }^{[69,70]}$. Colibactin warhead possessed the ability to directly bind to the duplex DNA with Spiro bicyclic structure. The bind will lead to depletion of the DNA mismatch repair system ${ }^{[71]}$. Another gut microbiota secreted substance with DNA damage ability to promote carcinogenesis indirectly ${ }^{[72]}$. Enterotoxigenic Bacteroides Fragilis encode B.fragilis metalloprotease toxin (BFT) which will induce diarrhea ${ }^{[73]}$. The secretion of enterotoxin BFT could also increase the polyamine metabolism and induce DNA damage ${ }^{[74,75]}$.

\subsection{Gut-liver cancer axis}

With respect to liver cancer, the second leading cause of cancer mortality worldwide, NAFLD has been implicated as the third cause of hepatocellular carcinoma (HCC) worldwide ${ }^{[76,77]}$. The gut-liver axis plays a pivotal role in the pathogenesis of NAFLD induced HCC. Communicates between intestine and liver are released bile acids and some bioactive mediators. Gut microbiota metabolize endogenous and exogenous substrates will translocate to the liver through the portal vein and influence liver functions ${ }^{[78]}$. Diet could cause gut dysbiosis which subsequently leads to overproduction of volatile organic compounds, mitochondrial enzymes, and damage to the liver ${ }^{[79]}$. Another example is related to deoxycholic acid (DCA), an obesity-induced gut microbial metabolite. It is reported that hepatic translocation of DCA will induce the senescence of hepatic stellate cells (HSCs) ${ }^{[80]}$. When it comes to alcoholic liver disease, which may also lead to hepatocellular carcinoma. Excessive intake of alcohol greatly hurt the gut barrier, influence its permeability and alter microbiota composition ${ }^{[81]}$. It is reported that chronic ethanol will affect several microbiotas and increase bacterial translocation, leading to the dysfunction of immune system ${ }^{[82]}$.

\subsection{Gut-Biliary tract cancer axis}

In some countries of eastern Asia, cholangiocarcinoma (CCA) was related to the infection of liver flukes Opisthorchis, as well as Clonorchis. Nevertheless, expect from these endemic regions, the main cause of CCA attributes to the chronic inflammation of bile ducts epithelium. This characteristic in common indicate that the presence of gut microbiota metabolites and its influence on immune system may participate in the inflamtory process. Previous researches in animal models have shown that infection with Opisthorchis changes the gut microbiota both in the bile and in intestinal tract, resulting in several bacteria colonization, such as Lachnospiraceae, Ruminococcaceae and Lactobacillaceae families. Also, the community was changed with the loss of Porphyromonadaceae, Erysipelotrichaceae and Eubacteriaceae ${ }^{[83]}$. What's more, H. pylori, a common pathogen in gastrointestinal morbidity, was also reported to induce hepatobiliary diseases ${ }^{[84]}$.

\subsection{Gut-lung cancer axis}

Recently, the function of gut microbiota in lung cancer attracted focus. The gut-lung axis mainly indicates the inflammation of blood vessels caused by translocation of bacteria and bacterial products across the gastrointestinal tract barrier ${ }^{[85]}$. Chronic lung diseases such as COPD, asthma and cancer usually occur along with gastrointestinal disorders. The most mutual hypothesis is that gut microbiota influence lung immunity. The intestinal microbiota is broadly protective against respiratory infection ${ }^{[86]}$. A study also found that segmented filamentous bacteria (SFB) play an important role in immunocompetent adaptive protection against $\mathrm{S}$. pneumoniae infection. However, SFB could expand dual T cell receptor (TCR)-expressing Th17 cells and bring out autoimmunity ${ }^{[87]}$. The induced autoimmunity may elevate IL-6 and IL-8 during inflammatory stress. IL-6 and IL-8 may impact on lung epithelial cells resulting in involved in tumorigenesis through stimulation of NF- $\square$ B-1 pathway ${ }^{[88]}$. Furthermore, IL-6 and 
IL-8 may also act in proliferation, migration, and invasion pf lung cancer since they were found over expressed in premalignant or senescent lung cancer cells.

\subsection{Gut-breast cancer axis}

Gut microbiota may influence estrogen levels to enhance the risk of breast cancer since they play an important role of in modulation of systemic estrogens ${ }^{[89]}$. Other than metabolism of oestrogens ${ }^{[90]}$, microbiota affects the enterohepatic circulation of estrogens. The possibility of breast cancer increased with heavy estrogen burden through enhanced reabsorption of free estrogen owing to estrobolome enrichment ${ }^{[1,92]}$. Apart from the influence on enterohepatic circulation of estrogens mentioned above, the gut-breast axis also can make a difference in breast cancer through the production of secondary bile acids as well ${ }^{[93]}$. Gut microbiota is responsible for converting primary bile acids to secondary bile acids, forming certain concentrations in human serum and breast tissue ${ }^{[94]}$. Lithocholic acid (LCA), a bacterial metabolite, is reported with the function of reducing proliferation and aggressiveness of breast cancer cells through inducing oxidative phosphorylation (OXPHOS), the trichloroacetic acid (TCA) cycle and antitumor immunity. The biosynthesis of LCA is regulated by gut microbiota such as Clostridium sordelli, Escherichia coli, Staphylococcus haemolyticus and so on ${ }^{[95]}$.

\subsection{Gut-haematopoiesis cancer axis}

In 2018, Nature reported that somatic mutations in tet methylcytosine dioxygenase 2 (TET2), which encodes an epigenetic modifier enzyme, drive the development of haematopoietic malignancies ${ }^{[96]}$. TET2 expression can be regulated by bacterial translocation and the subsequent interleukin-6 production in leukemia. Pre-leukaemic myeloproliferation occurs in only a fraction of Tet $2^{-/-}$mice and humans with TET2 mutations, suggesting that extrinsic non-cell-autonomous factors are required for disease onset. TET2 deficiency leads to increased selfrenewal of haematopoietic stem cells with a net developmental bias towards the myeloid lineage. However, It is reported that pre-leukaemic myeloproliferation can be reversed by antibiotic treatment and fail to develop in microbiota-free $\mathrm{Tet} 2^{-/-}$mice, which means the dysfunction of the small-intestinal barrier which caused bacterial translocation and increased interleukin- 6 production may have some positive effects in the progress in PMP ${ }^{[97]}$.

\subsection{Gut-brain cancer axis}

The gut-brain axis is included in complicated communication pathway including the central nervous system (CNS), neuroendocrine system, neuroimmune systems, autonomic nervous system, and the enteric nervous system. Gut microbiota can affect brain development and behaviors and conversely, brain signals can also regulate the gut microbiota ${ }^{[98]}$. Accumulating data indicate that the gut microbiota communicates with the CNS through gut-brain axis possibly through metabolites (SCFA), neuroendocrine axis (HPA Axis), vagus nerve and immune pathways and thereby influences brain function and behavior ${ }^{[99,100]}$. However, how gut microbiota influence brain cancer haven't been investigated. Some related study conveyed the gut microbiota can induce inflammatory in brain. For example, corticotrophin release factor 1 (CRFR1), a neuromodulator in the colon, is responsible for stress-induced permeability dysfunction and modulation of mucosal immune and inflammatory responses ${ }^{[101]}$.In nature 2018, Veit rothhammer, Davis M. Borucki et al. clearly put forward that intestinal microbial metabolites inhibiting CNS inflammation and affecting the pathogenicity of microglia and astrocytes ${ }^{[102]}$. However, our current understanding of how microbial affect vagal functions and subsequently signaling to the brain and how they communicate remains inadequate. Kristoffer L. Egerod, Natalia Petersen et al. determined the expression profile of all G protein-coupled receptors (GPCRs) in the vagal afferents of the mouse, especially vagal afferents who expressed NaV1.8 is richly innervating the entire GI mucosa ${ }^{[103]}$. Exposure of microbiota-free adult mice to a pathogen-free gut microbiota will decrease the permeability of blood-brain barrier and increase the expression of tight junction proteins ${ }^{[104]}$. Intestinal permeability is associated with systemic inflammation. Intestinal permeability may also induce the inflammation in the brain ${ }^{[104]}$. Last but not least, Camille Jacqueline and his team investigate that brain cancer could be associated with alterations in the intestinal microbial community for the first time. They 
identified a bacterial signature that cancerous larvae had a significantly lower relative abundance of Bacillaceae than individuals who did not develop the brain cancer ${ }^{[105]}$.

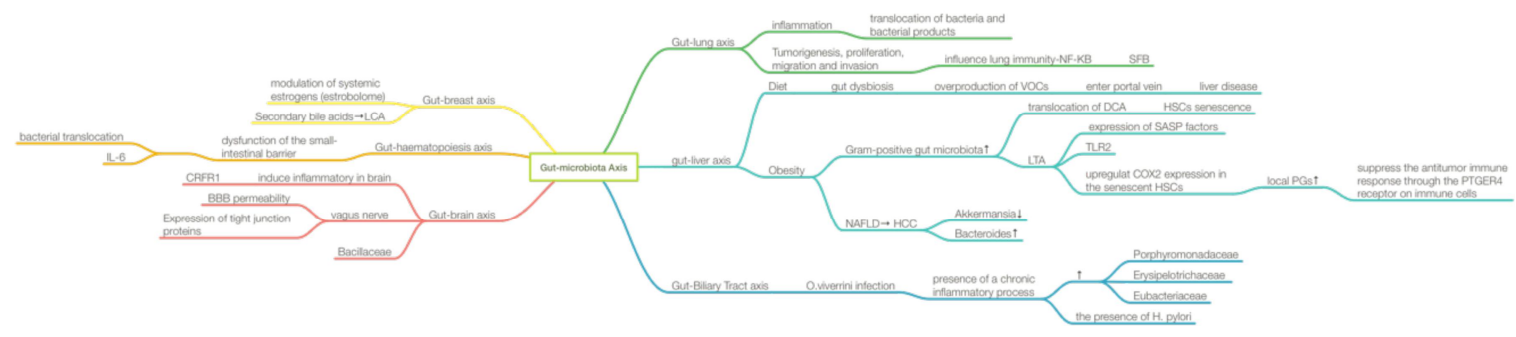

\section{IS IT CREDIBLE TO APPLY TO DIAGNOSE?}

Based on perturbation of these microbial communities might be associated with different types of cancer, we can suppose that bacterium could function as a marker for the development of cancer ${ }^{[106]}$. The current study demonstrated that the gut microbiome can be used as an effective tool in early screening for colorectal cancer. Streptococcus gallolyticus is proved to have the potential in indicating occult colonic adenomas and $\mathrm{CRC}^{[107]}$. Furthermore, gut microbiome showed its ability in distinguishing adenoma from carcinoma, with combination of several known demographical factors ${ }^{[108]}$. The effectiveness of gut microbiota test was proved similar to fecal occult blood test (FOBT) in colorectal cancer detection ${ }^{[109]}$. Although the gut microbiota could not singly indicate the occurrence of $\mathrm{CRC}$, such as Fusobacterium nucletum, the existence could somehow clue the highgrade dysplasia and a relatively unfavorable clinical outcome ${ }^{[110]}$. A large cohort reproved the association between Fusobacterium nucletum and $\mathrm{CRC}^{[111,112]}$. The poor prognosis may owe to the decreased $\mathrm{T}$-cell infiltration in the cancer sites $^{[113]}$.

\section{WHAT CAN WE DO AS TO MICROBIOTA TARGETED THERAPY?}

Gut microbiota also showed great potential in cancer therapy. Bacteroidetes and bifidobacteria was reported to enhance the treatment outcome through activating $\mathrm{T}$ cells ${ }^{[114]}$. They can strengthen the anti-tumor effect as a checkpoint blockade to inhibit tumor growth ${ }^{[115]}$ by targeting the CD $8+\mathrm{T}$ cells in the microenvironment ${ }^{[116]}$. Science also reported that gut microbiome may enhance the efficiency of PD-1 based immunotherapy against epithelial tumors including advanced melanoma, non-small cell lung cancer (NSCLC), and renal cell carcinoma $(\mathrm{RCC})^{[117]}$. Although the actual mechanism of PD-1 blockade enhancement still a mystery, the study put forward the possibility of combining microbiota and immunotherapy. Apart from immunotherapy, the combination of gut microbiota and chemical therapy also showed great significance. As a common antitumor drug, cisplatin could be applied to many kinds of cancer to mediate DNA damage of over-proliferated cells. However, fecal microbial transplantation (FMT) before chemotherapy could reduce the hurt of intestinal integrity caused by drug and bring down the possibility of systemic inflammation ${ }^{[18]}$. Faecalibacterium prausnitzii was found with the ability to upregulate the proliferation of $\mathrm{T}$ cells and reduce the number of IFN- $\gamma^{+} \mathrm{T}$ cells, creating an anti-inflammatory effect $^{[119]}$. Last but not least, not only probiotics but also some SCFA seem to be a safe approach with the conjunction of conventional treatment ${ }^{[120]}$.

Matrix metalloproteinases (MMPs), a family of zinc dependent endopeptidases, is reported to mediate inflammation, tissue remodeling, and tumorigenesis, among which MMP9 is the most unique one. MMP9 could only be detected up-regulated during inflammation and cancer with a protective role in tumorgenesis. On one hand, MMP9promotes the beneficial microbiota population and controls the ROS production. On the other, MMP9 restores the DNA damage via activation of the MMR pathway. This implies the use of targeted MMP9 inhibitors in CAC treatment therapies ${ }^{[121]}$. Bidisha Paul et.al found that genistein, an isoflavone found in soy, increased the os 
of breast tumor and reducing tumor growth through microbial alterations ${ }^{[122]}$. Alteration of microbiota abundance and/or composition could contribute to the therapy effect for breast tumor.

\section{WHERE CAN WE POLISH UP?}

\subsection{Biases according to different DNA extraction protocols}

DNA extraction of fecal samples in different protocols might introduce bias into the composition of gut microbiota $^{[123]}$. The protocols can be divided into physical ways and chemical methods. When the chemical methods were used, bacteria may show diverse resistance to different chemical agents ${ }^{[124]}$. The chemistry used may destroy the bacterial cell wall incompletely, leading to under- or over-estimation of different bacteria. Then, the composition of the gut microbiota might be improperly evaluated. Since there is no "gold standard" method for DNA extraction, some investigators prefer to combining several extraction methods to present the true community of gut microbota ${ }^{[125]}$.

\subsection{Lack of cohort studies with prospective design}

Majority of studies were based on mouse models and the researches couldn't distinguish whether the microbiota is the cause or the result of carcinogenesis ${ }^{[126]}$. Last but not least, most of the study focused on the microbiota composition by $16 \mathrm{~S}$ rRNA sequencing. Very few is known regarding the function of gut microbiota. Meta-genomic or meta-transcriptomic studies are in need. Functional analysis of gut microbiota would not only help us understanding the underlying mechanism for the link of bacteria and cancer, they may also help explain the inconsistent findings across studies.

\subsection{Other deficiency and future questions}

There remains a great deal of problems to solve such as the distinguish between "good" and "bad" bacteria, the detailed cellular mechanisms that are orchestrated by the gut microbiota and manipulation of the gut microbiota for benefit.

\section{CONCLUSION}

The mutualistic relationship between the host and gut microbiota favors mutual growth, survival and wellbeing. Despite numerous time and plenty development in understanding the composition of gut microbiota, many mechanisms remain uncovered. Nevertheless, there is no denying the fact that gut microbiota impacts human health and disease through different axis, and the promising aspect worth studying and application. Notably, prohibit the abuse of antibiotics must be enforced in that emerging data reveal that antibiotics can detrimentally regulate microflora ${ }^{[127]}$. With the application and integration of different types of data, the mechanism of how act in normal physiological activities and tumor formation will be explored. More importantly, gut microbiota-based therapy will be invented with the discovery of the mechanism. To conclude, more evidence is required in explicit the mechanism of gut microbiota in cancer environment, as well as more clinical practice in cancer therapy.

\section{COMPETING INTERESTS}

The authors declare no competing interests.

\section{REFERENCE}

[1] Jorth, P. et al. Metatranscriptomics of the human oral microbiome during health and disease. mBio 5, e0101201014, doi:10.1128/mBio.01012-14 (2014).

[2] Villanueva-Millán, M. J., Pérez-Matute, P. \& Oteo, J. A. Gut microbiota: a key player in health and disease. A 
review focused on obesity. $J$ Physiol Biochem 71, 509-525, doi:10.1007/s13105-015-0390-3 (2015).

[3] Tandon, D. et al. A prospective randomized, double-blind, placebo-controlled, dose-response relationship study to investigate efficacy of fructo-oligosaccharides (FOS) on human gut microflora. Scientific reports $\mathbf{9}$, 5473-5473, doi:10.1038/s41598-019-41837-3 (2019).

[4] Davenport, E. R. et al. The human microbiome in evolution. BMC Biol 15, 127-127, doi:10.1186/s12915-0170454-7 (2017).

[5] Hanahan, D. \& Weinberg, R. A. Hallmarks of cancer: the next generation. Cell 144, 646-674, doi:10.1016/ j.cell.2011.02.013 (2011).

[6] Talib, W. H. Melatonin and Cancer Hallmarks. Molecules (Basel, Switzerland) 23, doi:10.3390/molecules23030518 (2018).

[7] Consortium, H. M. P. Structure, function and diversity of the healthy human microbiome. Nature 486, 207214, doi:10.1038/nature11234 (2012).

[8] Qin, J. et al. A human gut microbial gene catalogue established by metagenomic sequencing. Nature 464, 5965, doi:10.1038/nature08821 (2010).

[9] Venkatesh, M. et al. Symbiotic bacterial metabolites regulate gastrointestinal barrier function via the xenobiotic sensor PXR and Toll-like receptor 4. Immunity 41, 296-310, doi:10.1016/j.immuni.2014.06.014 (2014).

[10] Sartor, R. B. Microbial influences in inflammatory bowel diseases. Gastroenterology 134, 577-594, doi:10.1053/j.gastro.2007.11.059 (2008).

[11] Zaibi, M. S. et al. Roles of GPR41 and GPR43 in leptin secretory responses of murine adipocytes to short chain fatty acids. FEBS letters 584, 2381-2386, doi:10.1016/j.febslet.2010.04.027 (2010).

[12] Shen, J., Obin, M. S. \& Zhao, L. The gut microbiota, obesity and insulin resistance. Molecular aspects of medicine 34, 39-58, doi:10.1016/j.mam.2012.11.001 (2013).

[13] Samuel, B. S. et al. Effects of the gut microbiota on host adiposity are modulated by the short-chain fatty-acid binding G protein-coupled receptor, Gpr41. Proceedings of the National Academy of Sciences of the United States of America 105, 16767-16772, doi:10.1073/pnas.0808567105 (2008).

[14] Magwira, C. A. et al. Diversity of faecal oxalate-degrading bacteria in black and white South African study groups: insights into understanding the rarity of urolithiasis in the black group. Journal of applied microbiology 113, 418-428, doi:10.1111/j.1365-2672.2012.05346.x (2012).

[15] Silk, D. B., Grimble, G. K. \& Rees, R. G. Protein digestion and amino acid and peptide absorption. The Proceedings of the Nutrition Society 44, 63-72 (1985).

[16] Neis, E. P., Dejong, C. H. \& Rensen, S. S. The role of microbial amino acid metabolism in host metabolism. Nutrients 7, 2930-2946, doi:10.3390/nu7042930 (2015).

[17] Thomas, C. M. et al. Histamine derived from probiotic Lactobacillus reuteri suppresses TNF via modulation of PKA and ERK signaling. PloS one 7, e31951, doi:10.1371/journal.pone.0031951 (2012).

[18] De Biase, D. \& Pennacchietti, E. Glutamate decarboxylase-dependent acid resistance in orally acquired bacteria: function, distribution and biomedical implications of the gadBC operon. Molecular microbiology 86, 770-786, doi:10.1111/mmi.12020 (2012).

[19] Tatullo, M. et al. Antioxidant and Antitumor Activity of a Bioactive Polyphenolic Fraction Isolated from the Brewing Process. Scientific reports 6, 36042, doi:10.1038/srep36042 (2016).

[20] Wang, D. et al. Role of intestinal microbiota in the generation of polyphenol-derived phenolic acid mediated attenuation of Alzheimer's disease beta-amyloid oligomerization. Molecular nutrition \& food research 59 , 1025-1040, doi:10.1002/mnfr.201400544 (2015).

[21] Duenas, M. et al. A survey of modulation of gut microbiota by dietary polyphenols. BioMed research international 2015, 850902, doi:10.1155/2015/850902 (2015).

[22] Ozdal, T. et al. The Reciprocal Interactions between Polyphenols and Gut Microbiota and Effects on Bioaccessibility. Nutrients 8, 78, doi:10.3390/nu8020078 (2016). 
[23] Goto, Y. et al. Innate lymphoid cells regulate intestinal epithelial cell glycosylation. Science (New York, N.Y.) 345, 1254009, doi:10.1126/science.1254009 (2014).

[24] Winston, J. A. \& Theriot, C. M. Impact of microbial derived secondary bile acids on colonization resistance against Clostridium difficile in the gastrointestinal tract. Anaerobe 41, 44-50, doi:10.1016/ j.anaerobe.2016.05.003 (2016).

[25] Russo, R. et al. Gut-brain Axis: Role of Lipids in the Regulation of Inflammation, Pain and CNS Diseases. Current medicinal chemistry 25, 3930-3952, doi:10.2174/0929867324666170216113756 (2018).

[26] Bieberich, E. It's a lipid's world: bioactive lipid metabolism and signaling in neural stem cell differentiation. Neurochemical research 37, 1208-1229, doi:10.1007/s11064-011-0698-5 (2012).

[27] Hooper, L. V. et al. Molecular analysis of commensal host-microbial relationships in the intestine. Science (New York, N.Y.) 291, 881-884, doi:10.1126/science.291.5505.881 (2001).

[28] Costantini, L., Molinari, R., Farinon, B. \& Merendino, N. Impact of Omega-3 Fatty Acids on the Gut Microbiota. International journal of molecular sciences 18, doi:10.3390/ijms18122645 (2017).

[29] Wallace, B. D. et al. Alleviating cancer drug toxicity by inhibiting a bacterial enzyme. Science (New York, N.Y.) 330, 831-835, doi:10.1126/science.1191175 (2010).

[30] Kamada, N., Chen, G. Y., Inohara, N. \& Nunez, G. Control of pathogens and pathobionts by the gut microbiota. Nature immunology 14, 685-690, doi:10.1038/ni.2608 (2013).

[31] Thaiss, C. A., Zmora, N., Levy, M. \& Elinav, E. The microbiome and innate immunity. Nature 535, 65-74, doi:10.1038/nature18847 (2016).

[32] Purchiaroni, F. et al. The role of intestinal microbiota and the immune system. European review for medical and pharmacological sciences 17, 323-333 (2013).

[33] Balmer, M. L. et al. Microbiota-derived compounds drive steady-state granulopoiesis via MyD88/TICAM signaling. Journal of immunology (Baltimore, Md. : 1950) 193, 5273-5283, doi:10.4049/jimmunol.1400762 (2014).

[34] Khosravi, A. et al. Gut microbiota promote hematopoiesis to control bacterial infection. Cell host \& microbe 15, 374-381, doi:10.1016/j.chom.2014.02.006 (2014).

[35] Bain, C. C. et al. Constant replenishment from circulating monocytes maintains the macrophage pool in the intestine of adult mice. Nature immunology 15, 929-937, doi:10.1038/ni.2967 (2014).

[36] Sawa, S. et al. RORgammat+ innate lymphoid cells regulate intestinal homeostasis by integrating negative signals from the symbiotic microbiota. Nature immunology 12, 320-326, doi:10.1038/ni.2002 (2011).

[37] Kruglov, A. A. et al. Nonredundant function of soluble LTalpha3 produced by innate lymphoid cells in intestinal homeostasis. Science (New York, N.Y.) 342, 1243-1246, doi:10.1126/science.1243364 (2013).

[38] Kelly, C. J. et al. Crosstalk between Microbiota-Derived Short-Chain Fatty Acids and Intestinal Epithelial HIF Augments Tissue Barrier Function. Cell host \& microbe 17, 662-671, doi:10.1016/j.chom.2015.03.005 (2015).

[39] Guilloteau, P. et al. From the gut to the peripheral tissues: the multiple effects of butyrate. Nutrition research reviews 23, 366-384, doi:10.1017/s0954422410000247 (2010).

[40] Paolella, G. et al. Gut-liver axis and probiotics: their role in non-alcoholic fatty liver disease. World journal of gastroenterology 20, 15518-15531, doi:10.3748/wjg.v20.i42.15518 (2014).

[41] Woting, A. \& Blaut, M. The Intestinal Microbiota in Metabolic Disease. Nutrients 8, 202, doi:10.3390/ nu8040202 (2016).

[42] Cummings, J. H. \& Macfarlane, G. T. The control and consequences of bacterial fermentation in the human colon. The Journal of applied bacteriology 70, 443-459 (1991).

[43] Kimura, I. et al. Short-chain fatty acids and ketones directly regulate sympathetic nervous system via G protein-coupled receptor 41 (GPR41). Proceedings of the National Academy of Sciences of the United States of America 108, 8030-8035, doi:10.1073/pnas.1016088108 (2011).

[44] Gasperotti, M. et al. Fate of microbial metabolites of dietary polyphenols in rats: is the brain their target 
destination? ACS chemical neuroscience 6, 1341-1352, doi:10.1021/acschemneuro.5b00051 (2015).

[45] Szwajgier, D., Borowiec, K. \& Pustelniak, K. The Neuroprotective Effects of Phenolic Acids: Molecular Mechanism of Action. Nutrients 9, doi:10.3390/nu9050477 (2017).

[46] Gerard, P. Metabolism of cholesterol and bile acids by the gut microbiota. Pathogens (Basel, Switzerland) 3 , 14-24, doi:10.3390/pathogens3010014 (2013).

[47] Dai, Z. L. et al. L-Glutamine regulates amino acid utilization by intestinal bacteria. Amino acids 45, 501-512, doi:10.1007/s00726-012-1264-4 (2013).

[48] Dai, Z. L., Wu, G. \& Zhu, W. Y. Amino acid metabolism in intestinal bacteria: links between gut ecology and host health. Frontiers in bioscience (Landmark edition) 16, 1768-1786 (2011).

[49] Rhee, S. H., Pothoulakis, C. \& Mayer, E. A. Principles and clinical implications of the brain-gut-enteric microbiota axis. Nature reviews. Gastroenterology \& hepatology 6, 306-314, doi:10.1038/nrgastro.2009.35 (2009).

[50] Lyte, M. Microbial endocrinology in the microbiome-gut-brain axis: how bacterial production and utilization of neurochemicals influence behavior. PLoS pathogens 9, e1003726, doi:10.1371/journal.ppat.1003726 (2013).

[51] Wang, B. et al. Luminal administration ex vivo of a live Lactobacillus species moderates mouse jejunal motility within minutes. FASEB journal : official publication of the Federation of American Societies for Experimental Biology 24, 4078-4088, doi:10.1096/fj.09-153841 (2010).

[52] Matsumoto, M. et al. Cerebral low-molecular metabolites influenced by intestinal microbiota: a pilot study. Frontiers in systems neuroscience 7, 9, doi:10.3389/fnsys.2013.00009 (2013).

[53] Lee, C. Y. \& Abizaid, A. The gut-brain-axis as a target to treat stress-induced obesity. Frontiers in endocrinology 5, 117, doi:10.3389/fendo.2014.00117 (2014).

[54] Marchesi, J. R. et al. The gut microbiota and host health: a new clinical frontier. Gut 65, 330-339, doi:10.1136/gutjnl-2015-309990 (2016).

[55] Sender, R., Fuchs, S. \& Milo, R. Revised Estimates for the Number of Human and Bacteria Cells in the Body. PLoS biology 14, e1002533, doi:10.1371/journal.pbio.1002533 (2016).

[56] Arnold, M. et al. Global patterns and trends in colorectal cancer incidence and mortality. Gut 66, 683-691, doi:10.1136/gutjnl-2015-310912 (2017).

[57] Claesson, M. J. et al. Composition, variability, and temporal stability of the intestinal microbiota of the elderly. Proceedings of the National Academy of Sciences of the United States of America 108 Suppl 1, 45864591, doi:10.1073/pnas.1000097107 (2011).

[58] Claesson, M. J. et al. Gut microbiota composition correlates with diet and health in the elderly. Nature 488, 178-184, doi:10.1038/nature11319 (2012).

[59] David, L. A. et al. Diet rapidly and reproducibly alters the human gut microbiome. Nature 505, 559-563, doi:10.1038/nature12820 (2014).

[60] Polk, D. B. \& Peek, R. M., Jr. Helicobacter pylori: gastric cancer and beyond. Nature reviews. Cancer 10, 403-414, doi:10.1038/nrc2857 (2010).

[61] Crosbie, E. J., Einstein, M. H., Franceschi, S. \& Kitchener, H. C. Human papillomavirus and cervical cancer. Lancet (London, England) 382, 889-899, doi:10.1016/s0140-6736(13)60022-7 (2013).

[62] Zheng, R., Zeng, H., Zhang, S., Chen, T. \& Chen, W. National estimates of cancer prevalence in China, 2011. Cancer letters 370, 33-38, doi:10.1016/j.canlet.2015.10.003 (2016).

[63] Chen, W. et al. Cancer statistics in China, 2015. CA: a cancer journal for clinicians 66, 115-132, doi:10.3322/ caac.21338 (2016).

[64] Coker, O. O. et al. Enteric fungal microbiota dysbiosis and ecological alterations in colorectal cancer. Gut, doi:10.1136/gutjnl-2018-317178 (2018).

[65] Feng, Q. et al. Gut microbiome development along the colorectal adenoma-carcinoma sequence. Nature communications 6, 6528, doi:10.1038/ncomms 7528 (2015). 
[66] Nakatsu, G. et al. Gut mucosal microbiome across stages of colorectal carcinogenesis. Nature communications 6, 8727, doi:10.1038/ncomms9727 (2015).

[67] Grivennikov, S. I. et al. Adenoma-linked barrier defects and microbial products drive IL-23/IL-17-mediated tumour growth. Nature 491, 254-258, doi:10.1038/nature11465 (2012).

[68] He, Z. et al. Campylobacter jejuni promotes colorectal tumorigenesis through the action of cytolethal distending toxin. Gut, doi:10.1136/gutjnl-2018-317200 (2018).

[69] Cuevas-Ramos, G. et al. Escherichia coli induces DNA damage in vivo and triggers genomic instability in mammalian cells. Proceedings of the National Academy of Sciences of the United States of America 107, 11537-11542, doi:10.1073/pnas.1001261107 (2010).

[70] Vizcaino, M. I. \& Crawford, J. M. The colibactin warhead crosslinks DNA. Nature chemistry 7, 411-417, doi:10.1038/nchem.2221 (2015).

[71] Maddocks, O. D., Scanlon, K. M. \& Donnenberg, M. S. An Escherichia coli effector protein promotes host mutation via depletion of DNA mismatch repair proteins. mBio 4, e00152-00113, doi:10.1128/mBio.0015213 (2013).

[72] Irrazabal, T., Belcheva, A., Girardin, S. E., Martin, A. \& Philpott, D. J. The multifaceted role of the intestinal microbiota in colon cancer. Molecular cell 54, 309-320, doi:10.1016/j.molcel.2014.03.039 (2014).

[73] Sears, C. L., Geis, A. L. \& Housseau, F. Bacteroides fragilis subverts mucosal biology: from symbiont to colon carcinogenesis. The Journal of clinical investigation 124, 4166-4172, doi:10.1172/jci72334 (2014).

[74] Snezhkina, A. V. et al. The Dysregulation of Polyamine Metabolism in Colorectal Cancer Is Associated with Overexpression of c-Myc and C/EBPbeta rather than Enterotoxigenic Bacteroides fragilis Infection. Oxidative medicine and cellular longevity 2016, 2353560, doi:10.1155/2016/2353560 (2016).

[75] Wu, S., Morin, P. J., Maouyo, D. \& Sears, C. L. Bacteroides fragilis enterotoxin induces c-Myc expression and cellular proliferation. Gastroenterology 124, 392-400, doi:10.1053/gast.2003.50047 (2003).

[76] McGuire, S. World Cancer Report 2014. Geneva, Switzerland: World Health Organization, International Agency for Research on Cancer, WHO Press, 2015. Advances in nutrition (Bethesda, Md.) 7, 418-419, doi:10.3945/an.116.012211 (2016).

[77] Michelotti, G. A., Machado, M. V. \& Diehl, A. M. NAFLD, NASH and liver cancer. Nature reviews. Gastroenterology \& hepatology 10, 656-665, doi:10.1038/nrgastro.2013.183 (2013).

[78] Starkel, P. \& Schnabl, B. Bidirectional Communication between Liver and Gut during Alcoholic Liver Disease. Seminars in liver disease 36, 331-339, doi:10.1055/s-0036-1593882 (2016).

[79] Reid, D. T. et al. Unique microbial-derived volatile organic compounds in portal venous circulation in murine non-alcoholic fatty liver disease. Biochimica et biophysica acta 1862, 1337-1344, doi:10.1016/ j.bbadis.2016.04.005 (2016).

[80] Loo, T. M. et al. Gut Microbiota Promotes Obesity-Associated Liver Cancer through PGE2-Mediated Suppression of Antitumor Immunity. Cancer discovery 7, 522-538, doi:10.1158/2159-8290.Cd-16-0932 (2017).

[81] Szabo, G. Gut-liver axis in alcoholic liver disease. Gastroenterology 148, 30-36, doi:10.1053/j.gastro.2014.10.042 (2015).

[82] Hendrikx, T. et al. Bacteria engineered to produce IL-22 in intestine induce expression of REG3G to reduce ethanol-induced liver disease in mice. Gut, doi:10.1136/gutjnl-2018-317232 (2018).

[83] Plieskatt, J. L. et al. Infection with the carcinogenic liver fluke Opisthorchis viverrini modifies intestinal and biliary microbiome. FASEB journal : official publication of the Federation of American Societies for Experimental Biology 27, 4572-4584, doi:10.1096/fj.13-232751 (2013).

[84] Itthitaetrakool, U. et al. Chronic Opisthorchis viverrini Infection Changes the Liver Microbiome and Promotes Helicobacter Growth. PloS one 11, e0165798, doi:10.1371/journal.pone.0165798 (2016).

[85] Schuijt, T. J. et al. The gut microbiota plays a protective role in the host defence against pneumococcal pneumonia. Gut 65, 575-583, doi:10.1136/gutjnl-2015-309728 (2016). 
[86] Tamburini, S. \& Clemente, J. C. Gut microbiota: Neonatal gut microbiota induces lung immunity against pneumonia. Nature reviews. Gastroenterology \& hepatology 14, 263-264, doi:10.1038/nrgastro.2017.34 (2017).

[87] Bradley, C. P. et al. Segmented Filamentous Bacteria Provoke Lung Autoimmunity by Inducing GutLung Axis Th17 Cells Expressing Dual TCRs. Cell host \& microbe 22, 697-704.e694, doi:10.1016/ j.chom.2017.10.007 (2017).

[88] Yuan, M., Meng, W., Liao, W. \& Lian, S. Andrographolide Antagonizes TNF-alpha-Induced IL-8 via Inhibition of NADPH Oxidase/ROS/NF-kappaB and Src/MAPKs/AP-1 Axis in Human Colorectal Cancer HCT116 Cells. Journal of agricultural and food chemistry 66, 5139-5148, doi:10.1021/acs.jafc.8b00810 (2018).

[89] McIntosh, F. M. et al. Phylogenetic distribution of genes encoding beta-glucuronidase activity in human colonic bacteria and the impact of diet on faecal glycosidase activities. Environmental microbiology 14, 18761887, doi:10.1111/j.1462-2920.2012.02711.x (2012).

[90] Fuhrman, B. J. et al. Associations of the fecal microbiome with urinary estrogens and estrogen metabolites in postmenopausal women. The Journal of clinical endocrinology and metabolism 99, 4632-4640, doi:10.1210/ jc. 2014-2222 (2014).

[91] Hullar, M. A., Burnett-Hartman, A. N. \& Lampe, J. W. Gut microbes, diet, and cancer. Cancer treatment and research 159, 377-399, doi:10.1007/978-3-642-38007-5_22 (2014).

[92] Modi, S. R., Collins, J. J. \& Relman, D. A. Antibiotics and the gut microbiota. The Journal of clinical investigation 124, 4212-4218, doi:10.1172/jci72333 (2014).

[93] Xie, G. et al. Dysregulated hepatic bile acids collaboratively promote liver carcinogenesis. International journal of cancer 139, 1764-1775, doi:10.1002/ijc.30219 (2016).

[94] Ridlon, J. M., Kang, D. J. \& Hylemon, P. B. Bile salt biotransformations by human intestinal bacteria. Journal of lipid research 47, 241-259, doi:10.1194/j1r.R500013-JLR200 (2006).

[95] Miko, E. et al. Lithocholic acid, a bacterial metabolite reduces breast cancer cell proliferation and aggressiveness. Biochimica et biophysica acta. Bioenergetics 1859, 958-974, doi:10.1016/j.bbabio.2018.04.002 (2018).

[96] Jan, M., Ebert, B. L. \& Jaiswal, S. Clonal hematopoiesis. Seminars in hematology 54, 43-50, doi:10.1053/ j.seminhematol.2016.10.002 (2017).

[97] Meisel, M. et al. Microbial signals drive pre-leukaemic myeloproliferation in a Tet2-deficient host. Nature 557, 580-584, doi:10.1038/s41586-018-0125-z (2018).

[98] Bailey, M. T. et al. Exposure to a social stressor alters the structure of the intestinal microbiota: implications for stressor-induced immunomodulation. Brain, behavior, and immunity 25, 397-407, doi:10.1016/ j.bbi.2010.10.023 (2011).

[99] Cryan, J. F. \& Dinan, T. G. Mind-altering microorganisms: the impact of the gut microbiota on brain and behaviour. Nature reviews. Neuroscience 13, 701-712, doi:10.1038/nrn3346 (2012).

[100]Cussotto, S., Sandhu, K. V., Dinan, T. G. \& Cryan, J. F. The Neuroendocrinology of the MicrobiotaGut-Brain Axis: A Behavioural Perspective. Frontiers in neuroendocrinology 51, 80-101, doi:10.1016/ j.yfrne.2018.04.002 (2018).

[101]Larauche, M., Kiank, C. \& Tache, Y. Corticotropin releasing factor signaling in colon and ileum: regulation by stress and pathophysiological implications. Journal of physiology and pharmacology : an official journal of the Polish Physiological Society 60 Suppl 7, 33-46 (2009).

[102] Rothhammer, V. et al. Microglial control of astrocytes in response to microbial metabolites. Nature 557, 724728, doi:10.1038/s41586-018-0119-x (2018).

[103]Egerod, K. L. et al. Profiling of G protein-coupled receptors in vagal afferents reveals novel gut-to-brain sensing mechanisms. Molecular metabolism 12, 62-75, doi:10.1016/j.molmet.2018.03.016 (2018).

[104]Braniste, V. et al. The gut microbiota influences blood-brain barrier permeability in mice. Science 
translational medicine 6, 263ra158, doi:10.1126/scitranslmed.3009759 (2014).

[105] Jacqueline, C. et al. Can intestinal microbiota be associated with non-intestinal cancers? Scientific reports 7 , 12722, doi:10.1038/s41598-017-11644-9 (2017).

[106]Aviles-Jimenez, F., Yu, G., Torres-Poveda, K., Madrid-Marina, V. \& Torres, J. On the Search to Elucidate the Role of Microbiota in the Genesis of Cancer: The Cases of Gastrointestinal and Cervical Cancer. Archives of medical research 48, 754-765, doi:10.1016/j.arcmed.2017.11.008 (2017).

[107]Boleij, A., van Gelder, M. M., Swinkels, D. W. \& Tjalsma, H. Clinical Importance of Streptococcus gallolyticus infection among colorectal cancer patients: systematic review and meta-analysis. Clinical infectious diseases : an official publication of the Infectious Diseases Society of America 53, 870-878, doi:10.1093/cid/cir609 (2011).

[108]Zackular, J. P., Rogers, M. A., Ruffin, M. T. t. \& Schloss, P. D. The human gut microbiome as a screening tool for colorectal cancer. Cancer prevention research (Philadelphia, Pa.) 7, 1112-1121, doi:10.1158/19406207.Capr-14-0129 (2014).

[109]Zeller, G. et al. Potential of fecal microbiota for early-stage detection of colorectal cancer. Molecular systems biology 10, 766, doi:10.15252/msb.20145645 (2014).

[110] Flanagan, L. et al. Fusobacterium nucleatum associates with stages of colorectal neoplasia development, colorectal cancer and disease outcome. European journal of clinical microbiology \& infectious diseases : official publication of the European Society of Clinical Microbiology 33, 1381-1390, doi:10.1007/s10096014-2081-3 (2014).

[111] Mima, K. et al. Fusobacterium nucleatum in colorectal carcinoma tissue and patient prognosis. Gut 65, 19731980, doi:10.1136/gutjnl-2015-310101 (2016).

[112] Viljoen, K. S., Dakshinamurthy, A., Goldberg, P. \& Blackburn, J. M. Quantitative profiling of colorectal cancer-associated bacteria reveals associations between fusobacterium spp., enterotoxigenic Bacteroides fragilis (ETBF) and clinicopathological features of colorectal cancer. PloS one 10, e0119462, doi:10.1371/ journal.pone.0119462 (2015).

[113] Mima, K. et al. Fusobacterium nucleatum and T Cells in Colorectal Carcinoma. JAMA oncology 1, 653-661, doi:10.1001/jamaoncol.2015.1377 (2015).

[114] Vetizou, M. et al. Anticancer immunotherapy by CTLA-4 blockade relies on the gut microbiota. Science (New York, N.Y.) 350, 1079-1084, doi:10.1126/science.aad1329 (2015).

[115] Tanabe, S. et al. Anti-inflammatory and Intestinal Barrier-protective Activities of Commensal Lactobacilli and Bifidobacteria in Thoroughbreds: Role of Probiotics in Diarrhea Prevention in Neonatal Thoroughbreds. Journal of equine science 25, 37-43, doi:10.1294/jes.25.37 (2014).

[116] Kaiser, J. Gut microbes shape response to cancer immunotherapy. Science (New York, N.Y.) 358, 573, doi:10.1126/science.358.6363.573 (2017).

[117] Routy, B. et al. Gut microbiome influences efficacy of PD-1-based immunotherapy against epithelial tumors. Science (New York, N.Y.) 359, 91-97, doi:10.1126/science.aan3706 (2018).

[118] Perales-Puchalt, A. et al. Frontline Science: Microbiota reconstitution restores intestinal integrity after cisplatin therapy. Journal of leukocyte biology 103, 799-805, doi:10.1002/jlb.5hi1117-446rr (2018).

[119] Rossi, O. et al. Faecalibacterium prausnitzii A2-165 has a high capacity to induce IL-10 in human and murine dendritic cells and modulates T cell responses. Scientific reports 6, 18507, doi:10.1038/srep18507 (2016).

[120]Martin, R. et al. Functional Characterization of Novel Faecalibacterium prausnitzii Strains Isolated from Healthy Volunteers: A Step Forward in the Use of F. prausnitzii as a Next-Generation Probiotic. Frontiers in microbiology 8, 1226, doi:10.3389/fmicb.2017.01226 (2017).

[121]Pujada, A. et al. Matrix metalloproteinase MMP9 maintains epithelial barrier function and preserves mucosal lining in colitis associated cancer. Oncotarget 8, 94650-94665, doi:10.18632/oncotarget.21841 (2017).

[122] Paul, B. et al. Impact of genistein on the gut microbiome of humanized mice and its role in breast tumor 
inhibition. PloS one 12, e0189756, doi:10.1371/journal.pone.0189756 (2017).

[123]Kashinskaya, E. N., Andree, K. B., Simonov, E. P. \& Solovyev, M. M. DNA extraction protocols may influence biodiversity detected in the intestinal microbiome: a case study from wild Prussian carp, Carassius gibelio. FEMS microbiology ecology 93, doi:10.1093/femsec/fiw240 (2017).

[124]von Wintzingerode, F., Gobel, U. B. \& Stackebrandt, E. Determination of microbial diversity in environmental samples: pitfalls of PCR-based rRNA analysis. FEMS microbiology reviews 21, 213-229, doi:10.1111/j.1574-6976.1997.tb00351.x (1997).

[125]Wen, C., He, Y., Xue, M., Liang, H. \& Dong, J. [Biases on community structure during DNA extraction of shrimp intestinal microbiota revealed by high-throughput sequencing] Wei sheng wu xue bao = Acta microbiologica Sinica 56, 130-142 (2016).

[126]Lertpiriyapong, K. et al. Gastric colonisation with a restricted commensal microbiota replicates the promotion of neoplastic lesions by diverse intestinal microbiota in the Helicobacter pylori INS-GAS mouse model of gastric carcinogenesis. Gut 63, 54-63, doi:10.1136/gutjnl-2013-305178 (2014).

[127]Nelson, M. H., Diven, M. A., Huff, L. W. \& Paulos, C. M. Harnessing the Microbiome to Enhance Cancer Immunotherapy. Journal of immunology research 2015, 368736, doi:10.1155/2015/368736 (2015). 